TITLE:

\title{
Ligand-to-Metal Charge-Transfer Dynamics in a Blue Copper Protein Plastocyanin: A Molecular Dynamics Study
}

$\operatorname{AUTHOR}(S)$ :

Ando, Koji

\section{CITATION:}

Ando, Koji. Ligand-to-Metal Charge-Transfer Dynamics in a Blue Copper Protein Plastocyanin: A Molecular Dynamics Study. The Journal of Physical Chemistry B 2007, 112(2): 250-256

ISSUE DATE:

2007-11-30

URL:

http://hdl.handle.net/2433/217064

\section{RIGHT:}

This document is the Accepted Manuscript version of a Published Work that appeared in final form in The 'Journal of Physical Chemistry B', copyright (c) American Chemical Society after peer review and technical editing by the publisher. To access the final edited and published work see http://dx.doi.org/10.1021/jp074822v.; This is not the published version. Please cite only the published version.; この論文は出版社版でありません。引用の際には出版社版をご確認ご 利用ください。 


\title{
Ligand-to-metal charge-transfer dynamics in a blue copper protein plastocyanin: A molecular dynamics study ${ }^{\dagger}$
}

\author{
Koji Ando \\ Department of Chemistry, Graduate School of Science, Kyoto University, Sakyo-ku, Kyoto 606-8502, Japan
}

\begin{abstract}
Equilibrium and non-equilibrium dynamics of a blue copper protein plastocyanin in oxidized state are studied by molecular dynamics (MD) simulation. Potential energy functions of the lowest seven electronic states, including ligand-to-metal charge-transfer (LMCT) and copper $\mathrm{d} \rightarrow \mathrm{d}$ excited states, were taken from our previous work (Ando, K. J. Phys. Chem. B 2004, 108, 3940) which employed $a b$ initio molecular orbital and density functional calculations on the active-site model. The equilibrium MD simulations in the ground state indicate that ligand motions coupled to transition from the ground state to the LMCT state are mostly represented by stretching and bending vibrations of the $\mathrm{Cu}-\mathrm{S}(\mathrm{Cys})$ distance, $\mathrm{N}_{\delta}(\mathrm{His})-\mathrm{Cu}-\mathrm{N}_{\delta}(\mathrm{His})$ angle, and $\mathrm{S}(\mathrm{Cys})-\mathrm{Cu}-$ $\left[\mathrm{N}_{\delta}(\mathrm{His})\right]_{2}$ trigonal pyramid structure. The non-equilibrium dynamics on the LMCT potential exhibit rapid decays in which surface crossings to the $\mathrm{d} \rightarrow \mathrm{d}$ and the first excited states occur in $70-80 \mathrm{fs}$. The crossing dynamics mostly correlate with cleavage of the $\mathrm{Cu}-\mathrm{S}(\mathrm{Cys})$ bond and associated response in the $\mathrm{N}_{\delta}(\mathrm{His})-\mathrm{Cu}-\mathrm{N}_{\delta}(\mathrm{His})$ moiety. The average dynamics of the vertical energy gap coordinates exhibit an overdamped decay with a recurrence oscillation in $500 \mathrm{fs}$ which shows clear coherence surviving after the ensemble averaging. This oscillation stems mostly from recoiling motion of the $\mathrm{N}_{\delta}(\mathrm{His})-\mathrm{Cu}-\mathrm{N}_{\delta}(\mathrm{His})$ part. The dynamics of the energy gaps after this coherent oscillation are randomized such that the ensemble average yields flat profiles along time, although each single trajectory exhibits fluctuations with amplitudes large enough to reach surface crossings. These indicate that the relaxation from the LMCT state first occurs via ballistic and coherent potential crossings in 70-80 fs and $500 \mathrm{fs}$, followed by thermally activated random transitions.
\end{abstract}

\section{INTRODUCTION}

Plastocyanins function as an electron carrier in photosynthesis in higher plants and algae $[1,2]$. They belong to the type I blue copper proteins which contain as the redox active-site a copper ion surrounded by a cysteine (Cys) and two histidine (His) ligands. The fourth ligand in plastocyanin is a methionine (Met) located in the axial direction of the distorted trigonal plane formed by the ion and the three ligands (Figure 1).

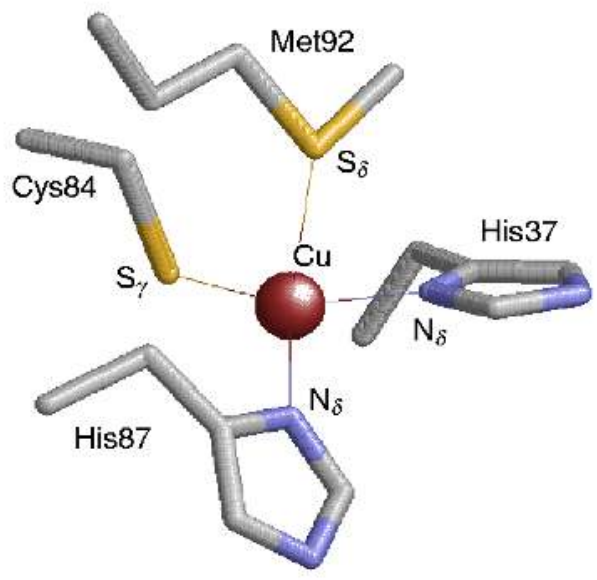

FIG. 1: The active-site structure of plastocyanin.

The ligand structure brings about strong optical absorption at $\sim 600 \mathrm{~nm}$, which is considered to originate from ligand-to-

†Part of the "James T. (Casey) Hynes Festschrift". metal charge-transfer (LMCT) transitions [3, 4]. Resonance Raman spectra [5, 6] indicate that the LMCT excitation is coupled to vibrational motions around the active-site in 350-450 $\mathrm{cm}^{-1}$ region. Ultrafast pump-probe experiments [7-9] have demonstrated that Fourier transformed time-resolved spectra correspond well with the resonance Raman peaks, and furthermore discovered [9] a lower frequency component of 33 $\mathrm{cm}^{-1}$. The pump-probe measurements also suggested that the excited LMCT state decays to the ground state via populating the intermediate copper $\mathrm{d} \rightarrow \mathrm{d}$ excited states in a few hundred femtoseconds. The spectroscopic investigations of the LMCT dynamics have been put forward with an aim to gain insights into the physiological electron transfer mechanism.

There also exist a number of molecular modeling studies on plastocyanin and related blue copper proteins [3, 10-20]. Most of them are either on the electronic structures around the equilibrium geometry, or molecular dynamics (MD) simulations in the ground state. In particular, to our knowledge, equilibrium and non-equilibrium dynamics of the energy gap coordinates (eq 2) involving the LMCT and other relevant excited states have not been analyzed previously.

In a recent study (paper I) [21], we have constructed potential energy functions of the lowest seven electronic states of plastocyanin in oxidized state by ab initio MCSCF (multiconfiguration self-consistent-field) and density functional calculations on an active-site model complex. We found that the fifth excited state possesses the LMCT character, though not in the ordinary form but is described mostly by an excitation from a $\mathrm{Cu}-\mathrm{S}$ (Cys) bonding orbital to a copper 3d orbital [22]. The potential energy surface in the LMCT state was found to be repulsive along the $\mathrm{Cu}-\mathrm{S}(\mathrm{Cys})$ distance (Figure 2) because of the loss of an electron from the bonding orbital. Also analyzed are the essential roles of the two His ligands to endow the LMCT character to the fifth excited state and to raise and modulate the energy separation between the LMCT and 


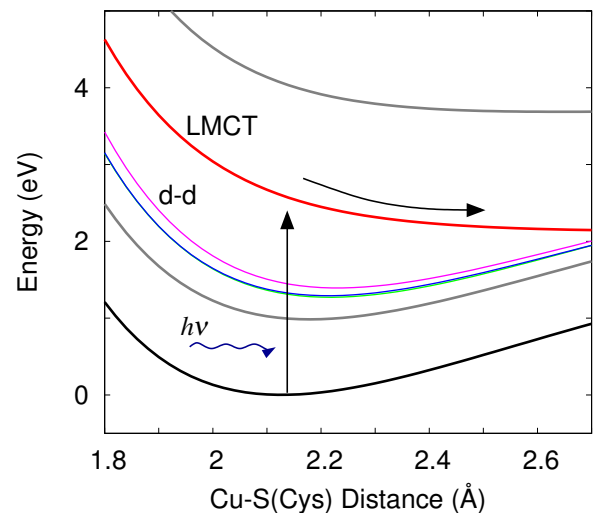

FIG. 2: Potential energy curves along the $\mathrm{Cu}-\mathrm{S}(\mathrm{Cys})$ distance in the model complex [21]. Note that these potentials are to be affected by the protein environment in the present MD study.

the lower states, which have been explained in terms of the electrostatic interactions with the negative net charges on the $\mathrm{N}_{\delta}$ (His) atoms [21].

With use of the potential functions developed in paper I, we carry out equilibrium and non-equilibrium MD simulations in this work. Our primary aim is to identify molecular motions coupled to the transition from the equilibrium ground state to the LMCT state, as well as the non-equilibrium dynamics induced by photoexcitation to the LMCT state. Explicit simulations of the non-adiabatic transition dynamics are beyond the scope of this article, but will be reported elsewhere with use of the mixed quantum-classical Liouville MD method [23, 24]. Nonetheless, the present results seem to be substantially informative of new insights into the molecular mechanism of the plastocyanin photodynamics.

\section{COMPUTATIONAL}

a. Potential Energy Functions. As noted above, the potential energy functions are taken from paper I in which the electronic structure calculations have been carried out on a model active-site complex consisting of a $\mathrm{Cu}$ (II) ion and truncated amino acid models of the four ligands, a $\mathrm{SCH}_{3}^{-}$, two imidazole rings, and a $\mathrm{S}\left(\mathrm{CH}_{3}\right)_{2}$. They are connected back to the amino acid side-chains after adjusting the atomic charges on the boundary (link) atoms to affirm consistent total charges for each residue. The resultant parameters are listed in Tables S1 and S2 in the Supporting Information. The other parameters and additional functions, such as the Morse potentials for the $\mathrm{Cu}-\mathrm{S}(\mathrm{Cys})$ bond, have been described in paper I. For the rest of the protein, the AMBER99 force-field [25] was employed. We used the flexible SPC model [26] for the solvent water.

b. Molecular Dynamics Simulations. The starting atomic coordinates for the MD simulation were taken from the Protein Data Bank (code 1BXU) from the X-ray crystallography on Synechococcus sp. PCC 7942 plastocyanin [27]. Hydrogen atoms were supplied such that aspartic and
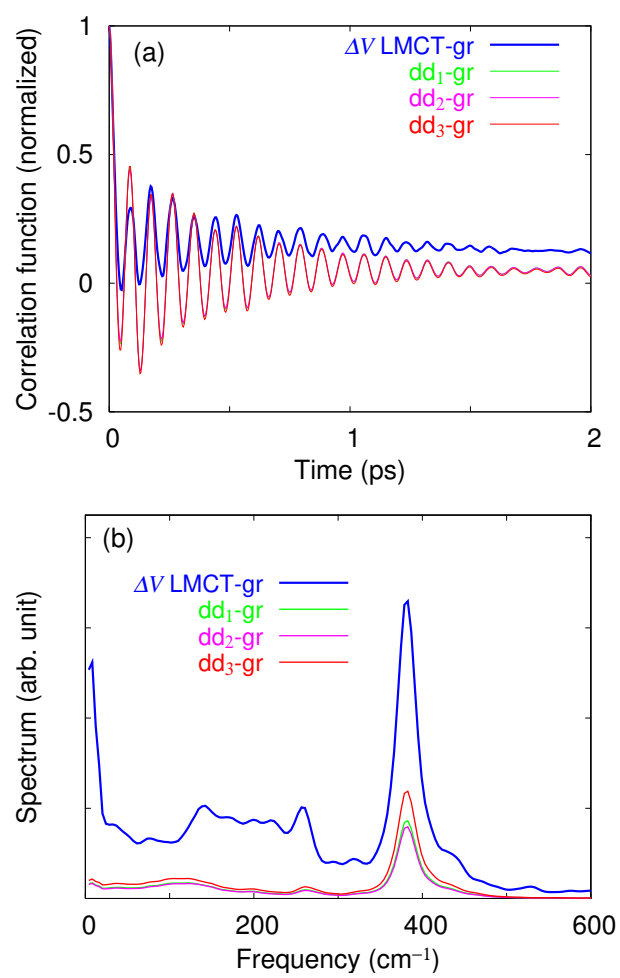

FIG. 3: Time correlation functions (a) and power spectra (b) of the energy gap coordinates eq 2 from the ground state to the LMCT and the $\mathrm{d} \rightarrow \mathrm{d}$ excited states, computed from the equilibrium simulation in the ground state. The statistical errors in the TCFs are smaller than \pm 0.03 .

glutamic acids have a negative charge, lysines and arginines have a positive charge, histidine-57 has a positive charge, and the $\mathrm{N}$ - and $\mathrm{C}$-terminal glutamines have a positive and negative charges. These residues locate in the surface region of the protein. The cysteine adjacent to the copper ion has a negative charge (more precisely, the $\mathrm{Cu}$ (II)-Cys moiety has a net positive charge), and all the other residues are assumed to be neutral. The total charge of the protein is thus -4 . We did not include counter ions to neutralize the simulation box for the reasons commented in Appendix A.

The protein was soaked in 3928 solvent waters in a cubic box of length $52.3 \AA$, which were determined so that the minimum distance between the solute protein and the box walls is greater than $9 \AA$. Also included are the 82 waters found in the X-ray crystallography. The periodic boundary condition was applied. The long-range non-bonded interactions were smoothly truncated with reference to the distance between the residue centers. The implementation of this non-conventional cut-off method is described in Appendix A. The cut-off distances are taken as $r_{\text {cut } 1}=18 \AA$ and $r_{\text {cut } 2}=1.1 r_{\text {cut } 1}$ such that the solute protein does not interact with its periodic images.

The classical equations of motion were integrated with use of the Suzuki-Trotter decomposition scheme [28]. The time steps for integration were 0.25 fs for bonded interactions and 2 fs for non-bonded interactions. For constant temperature sim- 
ulations, the Nosé thermostat [29] was applied with the time constant of 0.01 ps. We used the PEACH 3.8 software [30] for protein MD simulations, with minor modifications introduced to take account of the multiple electronic states and to implement the residue-center-based smooth cut-off (Appendix A).
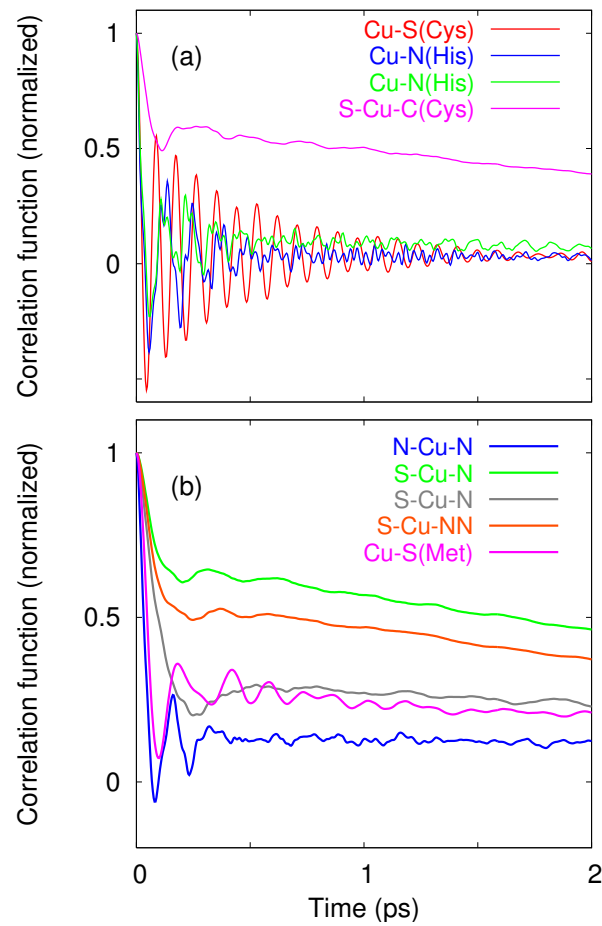

FIG. 4: Time correlation functions of the structural parameters (bond distances and angles) from the equilibrium simulation in the ground state. The statistical errors are smaller than \pm 0.04 for the bond distances TCFs and \pm 0.09 for the bond angles TCFs.

Before collecting the data, the system in the ground electronic state was first slowly heated up to $298 \mathrm{~K}$ in $210 \mathrm{ps}$, and then kept around $298 \mathrm{~K}$ for another 100 ps for equilibration. The equilibrium simulation in the ground state was carried out for 800 ps at $298 \mathrm{~K}$, from which the time correlation functions (TCFs) and the spectra in Figures 3-6 were computed [31]. We confirmed the convergence by comparing results from different simulation lengths. (Indeed, the results converged qualitatively in 400 ps.) The statistical errors in the TCFs are evaluated by the standard method [32] and are noted in the figure captions. In the course of the equilibrium simulation in the ground state, 400 configurations were sampled with random intervals longer than 1 ps [33]. The nonequilibrium dynamics in the LMCT state (Figures 7-9) were initiated from these sampled configurations and run under the micro-canonical condition. The statistical errors in the ensemble averages over the non-equilibrium trajectories were evaluated by assuming that the configurations sampled with the intervals longer than 3 ps are statistically independent.
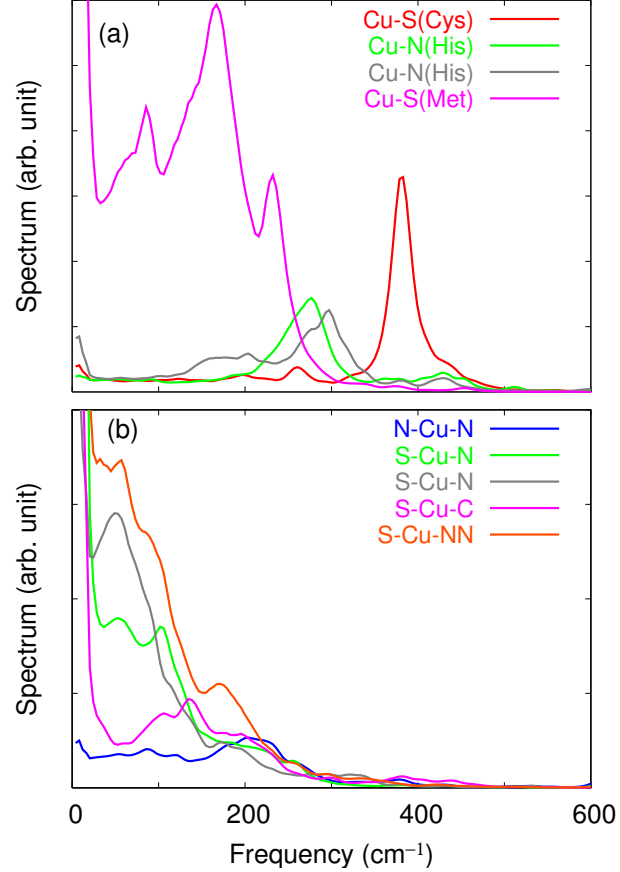

FIG. 5: Power spectra of the structural parameters corresponding to those in Figure 4.

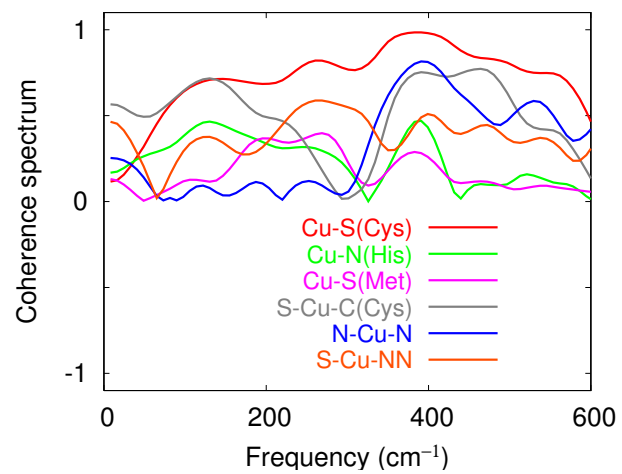

FIG. 6: Coherence spectra between the energy gap coordinate $\Delta V_{\mathrm{LMCT}-\text { ground }}$ and the structural parameters, computed from the equilibrium simulation in the ground state.

\section{RESULTS AND DISCUSSION}

\section{A. Equilibrium dynamics in the ground state}

We first examine the equilibrium properties in the ground state. Figure 3 a shows the normalized TCFs

$$
C_{i-j}(t)=\frac{\left\langle\delta \Delta V_{i-j}(0) \delta \Delta V_{i-j}(t)\right\rangle_{0}}{\left\langle\delta \Delta V_{i-j}(0)^{2}\right\rangle_{0}}
$$

of the vertical energy gap coordinates,

$$
\Delta V_{i-j}(t)=V_{i}(\boldsymbol{R}(t))-V_{j}(\boldsymbol{R}(t)),
$$


in which $V_{i}(\boldsymbol{R})$ is the total potential energy in the state $i$ at the nuclear configuration $\boldsymbol{R}$ of the entire system, $\delta \Delta V \equiv$ $\Delta V-\langle\Delta V\rangle_{0}$ is the fluctuation around the average, and $\langle\cdots\rangle_{0}$ denotes the equilibrium average in the ground state. The energy gap coordinates are the most appropriate projection of the many-dimensional interactions for description of non-adiabatic electronic transitions [34-37], since the surface crossing condition is simply and thoroughly represented by $\Delta V=0$. In the analysis of the equilibrium simulations, the reference state $j$ in eq 2 is the ground state.

It is seen in Figure 3a that the energy gap TCFs exhibit underdamped oscillatory decays. The oscillations correspond to the prominent peaks at $\sim 380 \mathrm{~cm}^{-1}$ in the Fourier transformed TCFs [38] in Figure $3 b$. The spectrum of the $\Delta V_{\mathrm{LMCT}-\text { ground }}$ also shows a broad structure in the $100-300 \mathrm{~cm}^{-1}$ region, which will be analyzed next. We also find that the energy gap dynamics are clearly different from the dynamics of the potential energies themselves, as demonstrated in Figures S1 and $\mathrm{S} 2$ in the Supporting Information.

The TCFs [39] and their spectra of the molecular structural parameters around the active-site are displayed in Figures 4 and 5. The structural parameters calculated in this work are the distances, angles, and torsional angles formed by the $\mathrm{Cu}$, $\mathrm{S}(\mathrm{Cys}), \mathrm{C}_{\beta}$ (Cys), two $\mathrm{N}_{\delta}$ (His), and $\mathrm{S}$ (Met) atoms, and the angle between the $\mathrm{Cu}-\mathrm{S}(\mathrm{Cys})$ vector and the bisector between the two $\mathrm{Cu}-\mathrm{N}_{\delta}(\mathrm{His})$ vectors which we call hereafter the 'pyramidal angle'.

We see in Figure 4 that the TCFs of the $\mathrm{Cu}-\mathrm{S}(\mathrm{Cys})$ and $\mathrm{Cu}-$ $\mathrm{N}_{\delta}$ (His) distances exhibit underdamped oscillatory decays. In particular, the former closely resembles the TCFs of the energy gaps in Figure 3a. However, the Fourier transformed spectra clearly indicate that the energy gap dynamics cannot be represented simply by the $\mathrm{Cu}-\mathrm{S}(\mathrm{Cys})$ stretching motion. We also see in Figure $4 \mathrm{~b}$ that the $\mathrm{Cu}-\mathrm{S}$ (Met) distance and the $\mathrm{N}_{\delta}$ (His)-Cu- $\mathrm{N}_{\delta}$ (His) angle show weakly damped oscillations, while the other angle parameters present overdamped decays with weak oscillations.

Comparison of the spectra in Figures $3 \mathrm{~b}$ and 5 seems to provide insights into the structural origin of the energy gap dynamics. However, the spectra are broad and mutually overlapping, and mere coincidence of the frequency does not necessarily determine the direct correlation. The analysis of the coherence and phase spectra [40, 41] (summarized in Appendix B) offers a useful tool to resolve the genuine correlations. As noted above, it is essential to explicitly examine the cross-correlations between the energy gap coordinate and the structural parameters. Figure 6 shows the coherence spectra between $\Delta V_{\mathrm{LMCT} \text {-ground }}$ and selected structural parameters which exhibit notable correlations. It clearly indicates that the $\mathrm{Cu}-\mathrm{S}(\mathrm{Cys})$ distance, $\mathrm{N}_{\delta}(\mathrm{His})-\mathrm{Cu}-\mathrm{N}_{\delta}(\mathrm{His})$ angle, and the $\mathrm{S}(\mathrm{Cys})-\mathrm{Cu}-\left[\mathrm{N}_{\delta}(\mathrm{His})\right]_{2}$ pyramidal angle constitute the principal components coupled to the transition from the ground state to the LMCT state. As has been suggested previously from the potential curves [21], the coupling of the $\mathrm{S}-\mathrm{Cu}-\mathrm{C}_{\beta}$ angle within the $\mathrm{Cys}$ and the $\mathrm{Cu}-\mathrm{N}$ (His) distance are also notable. The phase spectra (not shown) are confirmed to be negligibly small in the studied frequency region [42], indicating that the time lags in the correlated dynamics are insignificant.

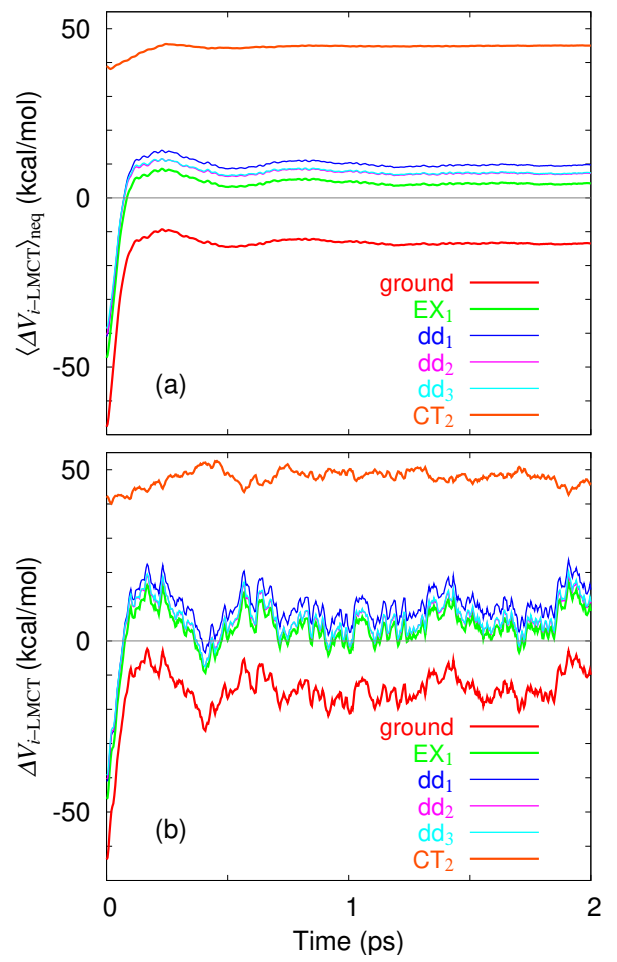

FIG. 7: Non-equilibrium dynamics of the energy gap coordinate $\Delta V_{i-\mathrm{LMCT}}$ on the LMCT potential, from an ensemble average (a) and a representative single trajectory (b). $\mathrm{EX}_{1}$ and $\mathrm{CT}_{2}$ denote the first and sixth excited states, respectively. The statistical errors in the averages are estimated to be smaller than $\pm 0.6 \mathrm{kcal} / \mathrm{mol}$.

\section{B. Non-equilibrium dynamics in the LMCT state}

We next examine the non-equilibrium dynamics initiated on the LMCT potential energy surface. As noted in section 2 , the starting configurations are sampled randomly with the intervals longer than $1 \mathrm{ps}$ in the course of the equilibrium simulation in the ground state. Figure 7a shows the ensemble averages of the energy gap dynamics measured from the LMCT state, $\left\langle\Delta V_{i-\mathrm{LMCT}}(t)\right\rangle_{\text {neq }}$, where $\langle\cdots\rangle_{\text {neq }}$ denotes the ensemble average over the non-equilibrium trajectories. As has been noted, the condition $\Delta V_{i-\mathrm{LMCT}}=0$, marked by the dotted horizontal axis in Figure 7, directly and thoroughly represents the surface crossings from the LMCT state to the other state. Since the nuclear tunnelings are reasonably assumed minor in this system, the non-adiabatic transitions would mostly occur at the surface crossings. Therefore, even though the nonadiabatic transitions are not explicitly simulated here, the dynamics of $\Delta V_{i-\mathrm{LMCT}}(t)$, together with the localized surfacehopping picture, will yield most relevant information about the molecular mechanism of the non-adiabatic transitions.

We observe in Figure 7a that the initial ballistic motion induces the surface crossings with the three $\mathrm{d} \rightarrow \mathrm{d}$ states and the first excited state in 70-80 fs time scale. They next show a recurrence oscillation in $\sim 500 \mathrm{fs}$. After these coherent motions, the averaged dynamics are almost damped to display flat time profiles. However, as illustrated by an example trajectory in 
Figure $7 b$, each single trajectory exhibit fluctuations with amplitudes large enough to induce a number of crossing events in the picoseconds time scale. To check the variance of this picture, six more randomly sampled trajectories are displayed in Figure S3 in the Supporting Information.

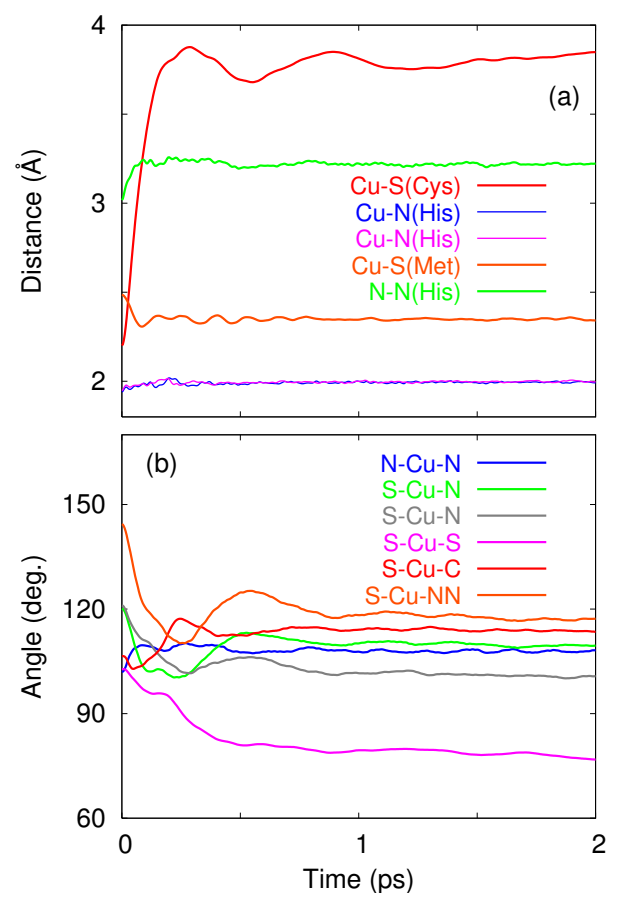

FIG. 8: Non-equilibrium dynamics on the LMCT potential of the structural parameters computed from the ensemble average over the trajectories. The statistical errors are estimated to be smaller than $\pm 0.06 \AA$ for the bond distances and $\pm 2 \mathrm{deg}$. for the bond angles.

We see in Figure 7 that the ground state remains below the LMCT state by more than $9 \mathrm{kcal} / \mathrm{mol}$, i.e., $\left\langle\Delta V_{\text {ground-LMCT }}(t)\right\rangle_{\text {neq }}<-9 \mathrm{kcal} / \mathrm{mol}$, suggesting that direct transitions from the LMCT to the ground state are not highly probable but the relaxation will occur via the intermediate $\mathrm{d} \rightarrow \mathrm{d}$ and the first excited states. It is also seen that the sixth excited state $\left(\mathrm{CT}_{2}\right.$ in the figure), which possesses a charge-transfer character [21], stays above the LMCT state by $\sim 40 \mathrm{kcal} / \mathrm{mol}$.

It would be assumed reasonably that the molecular motions pertaining to the transitions in the random thermal motions in the longer time region ( $>500 \mathrm{fs}$ ) are similar to those found in the equilibrium simulations. We thus focus on the initial ballistic and coherent motions discovered in the non-equilibrium simulations. Figure 8 shows the ensemble averaged dynamics of the structural parameters. The corresponding single trajectories are displayed in Figure S4 in the Supporting Information. As expected (Figure 2), the rapid initial motion of the energy gap appears to be closely correlated to the cleavage of the $\mathrm{Cu}-\mathrm{S}(\mathrm{Cys})$ bond. It is seen that the potential energy crossings in 70-80 fs occur at $\mathrm{Cu}-\mathrm{S}(\mathrm{Cys})=3.0-3.2 \AA$. This looks slightly longer than what had been anticipated simply from the potential curves of the model complex (Figure 2). The difference reflects the influences from the protein environment.

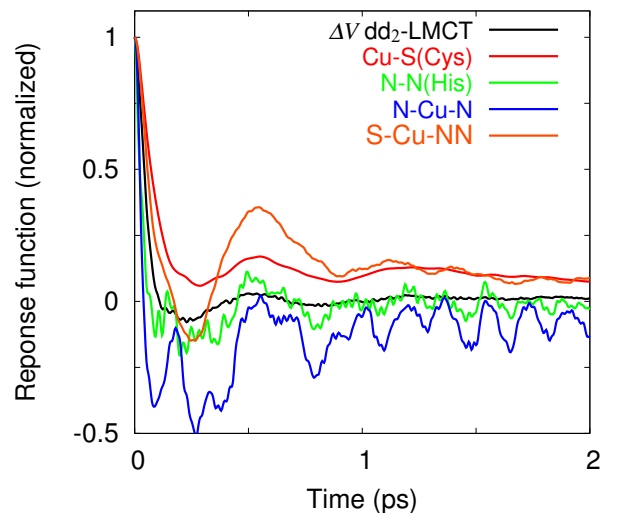

FIG. 9: Normalized response function eq 3 of selected parameters from Figures 7 and 8.

To further explore the correlation between the energetic and the structural responses, we examine in Figure 9 the normalized response functions defined for a parameter $A(t)$ by [43]

$$
\Phi(t)=\frac{\langle A(t)\rangle_{\text {neq }}-\langle A(\infty)\rangle_{\text {neq }}}{\langle A(0)\rangle_{\text {neq }}-\langle A(\infty)\rangle_{\text {neq }}} .
$$

It is suggested from Figure 9 that the coherent recurrence oscillation of the energy crossing dynamics is correlated with the recoiling responses of the $\mathrm{N}_{\delta}(\mathrm{His})-\mathrm{Cu}-\mathrm{N}_{\delta}(\mathrm{His})$ angle and the $\mathrm{S}(\mathrm{Cys})-\mathrm{Cu}-\left[\mathrm{N}_{\delta}(\mathrm{His})\right]_{2}$ pyramidal angle. Interestingly, these motions appear to be also associated closely with the initial rapid decay of the energy gap $\Delta V_{\mathrm{dd}-\mathrm{LMVT}}(t)$ in 70-80 fs.

\section{CONCLUDING REMARKS}

The principal results of this work are represented by Figures $3,6,7$ and 9. The equilibrium simulations in the ground state indicate that the $\mathrm{Cu}-\mathrm{S}(\mathrm{Cys})$ distance, $\mathrm{N}_{\delta}(\mathrm{His})-\mathrm{Cu}-\mathrm{N}_{\delta}(\mathrm{His})$ angle, and $\mathrm{S}(\mathrm{Cys})-\mathrm{Cu}-\left[\mathrm{N}_{\delta}(\mathrm{His})\right]_{2}$ pyramidal angle are the major coupling motions to the transition from the ground state to the LMCT state. These structural parameters are also most closely involved in the non-equilibrium dynamics initiated on the LMCT potential. The non-equilibrium simulations suggest that the relaxation from the LMCT state to the lower states first occurs via ballistic and coherent potential crossings in 70-80 fs and $500 \mathrm{fs}$ time scales, followed by thermally activated random transitions.

The simulated initial crossings in $70-80$ fs seem to be in accord with the ultrafast measurements which suggested that the excited LMCT state decays to the ground state via populating the intermediate $\mathrm{d} \rightarrow \mathrm{d}$ excited states in a few hundred femtoseconds [7, 8]. Moreover, the recoiling dynamics in 500 fs appears to correspond well with the damped oscillation discovered in the pump-probe analysis [9].

In order to advance the correspondence with experiments, we need to explicitly simulate the non-adiabatic transition dynamics [44]. To this end, mixed quantum-classical Liouville MD methods [23, 24, 45-48] will provide accurate pic- 
tures by taking appropriate account of the electronic coherence, though after much more elaborate computations than the independent-trajectories calculations such as the surfacehopping methods $[49,50]$. With the expected decoherence effects in the condensed phase, it would be likely that the latter methods are sufficient for this system. In this sense, a simpler approach, which deals with non-equilibrium average dynamics of reaction coordinates and thermal fluctuations around them [51], may be appropriately extended. It would be also intriguing to carry out direct QM/MM simulations $[52,53]$, which is however currently too demanding to achieve sufficient statistical samplings for the present system involving $\mathrm{d} \rightarrow \mathrm{d}$ and LMCT excited states. We envisage that these advanced calculations will add details to, but not essentially alter, the pictures emerged in this work.

While the physical chemistry of the LMCT dynamics is an interesting subject per se, more fundamental question should be around the physiological redox reactions. Since the $\mathrm{Cu}-$ $\mathrm{S}$ (Cys) bonding orbital will remain occupied in the reduced state [21], such large amplitude motions as observed in the non-equilibrium LMCT dynamics will be absent in the redox processes, but the other parts of the pictures from the present simulations are more likely to carry over. An interesting problem is therefore to clarify the common and distinct aspects between the LMCT photodynamics and the redox reactions. Works along this line will be reported elsewhere.

\section{Acknowledgments}

This work was partially supported by the Royal Society (G503/22211), EPSRC (GR/R60515), and KAKENHI (18031021, in Priority Area "Water and Biomolecules").

\section{Supporting Information Available:}

Tables of atomic charge parameters. Figures of the time correlation functions and their spectra for the energy gap coordinates and the ground state potential energy to the longer time and higher frequency ranges. Figures of the nonequilibrium dynamics of the energy gap coordinates from several randomly sampled trajectories. Figures of the nonequilibrium dynamics of the structural parameters from a representative single trajectory.

\section{Appendix A. Residue-center-based smooth potential cut-off}

The problems around the finite system size are inherent in condensed phase simulations. Periodic boundary conditions conveniently remove the boundary walls, but the imposed periodicity is not necessarily realistic for solvated proteins. The periodicity is enhanced with use of the Ewald sum method, which furthermore requires charge neutrality in the simulation box and therefore inclusion of counter ions around charged proteins. This may, however, result in unreasonably high concentration of the ions in the simulation box. These concerns led us to consider instead the potential cut-off methods. Nonetheless, it is well known that the cut-off methods suffer their own problems [54-56]. For example, simple abrupt cutoff induces instability of the energy. The atom-based cut-off also causes a charge neutrality (or consistency) problem for molecular pairs in the boundary region. To circumvent these problems, we have implemented a smooth cut-off method based on the residue centers. It would be intriguing to note here that similar motivation has led to recent examinations and developments [57] in the computation of pairwise electrostatic interactions in ionic crystals and simple polar liquids.

Let us denote by $v\left(r_{i j}\right)$ the bare potential between atoms $i$ and $j$ separated by a distance $r_{i j}$. We use subscripts $a$ and $b$ for central atoms in the amino acid residues (or solvent molecules) to which the atoms $i$ and $j$ belong. The central atoms are chosen, for example, as those closest to the center of mass of the residue. We introduce a smooth cut-off function $\sigma\left(r_{a b}\right)$ with parameters $r_{\text {cut } 1}$ and $r_{\text {cut } 2}$ by which the bare potential $v\left(r_{i j}\right)$ is scaled as

$$
u\left(r_{i j}\right)=\sigma\left(r_{a b}\right) \cdot v\left(r_{i j}\right) .
$$

The functional form of $\sigma\left(r_{a b}\right)$ should be such that it is unity in $r_{a b} \leq r_{\text {cut } 1}$, smoothly decays in $r_{\text {cut } 1}<r_{a b}<r_{\text {cut } 2}$, and vanishes in $r_{\text {cut } 2} \leq r_{a b}$. Our choice in this work is

$$
\sigma(\xi)=1-(10-\xi(15-6 \xi)) \xi^{3}
$$

in $0<\xi<1$, where we have introduced a dimensionless variable

$$
\xi=\frac{r_{a b}-r_{\text {cut } 1}}{r_{\text {cut } 2}-r_{\text {cut } 1}} .
$$

However, the following equations do not depend on the choice of $\sigma(\xi)$.

We assume that the total potential $U$ is a sum of the pair potentials

$$
U\left(x_{1}, x_{2}, \cdots\right)=\sum_{i<j} u\left(r_{i j}\right),
$$

where $x_{i}$ represent the Cartesian coordinates. The forces are then affected by the scaling factor $\sigma(\xi)$ such that for $i \neq a$,

$$
F_{i}=-\frac{\partial U}{\partial x_{i}}=-\sum_{j} \sigma(\xi) \frac{\partial v\left(r_{i j}\right)}{\partial x_{i}}
$$

For the central atoms we find

$$
F_{a}=-\frac{\partial U}{\partial x_{a}}=-\sum_{j} \sigma^{\prime}(\xi) \frac{\partial \xi}{\partial x_{a}} v\left(r_{a j}\right)-\sum_{j} \sigma(\xi) \frac{\partial v\left(r_{a j}\right)}{\partial x_{a}}
$$

in which $\sigma^{\prime}=d \sigma / d \xi$.

One of the merits of this method is that because the pair potentials are scaled by the common factor $\sigma(\xi)$ referring to the residue centers, the charge neutrality is always maintained for interactions between neutral residues. On the contrary, if a simple atom-based cut-off were used, the charge neutrality may be violated when the molecules lie across the cut-off boundary region, which may cause instability of Coulomb interactions. Similar argument applies to interactions involving charged residues. 


\section{Appendix B. Coherence and phase spectra}

The coherence spectrum [40, 41] is defined from the crossspectrum $S_{x y}(\omega)$ of two fluctuating variables $x(t)$ and $y(t)$ by

$$
\operatorname{Coh}(\omega)=\frac{\left|S_{x y}(\omega)\right|}{\sqrt{ } S_{x x}(\omega) S_{y y}(\omega)},
$$

in which $S_{x x}(\omega)$ and $S_{y y}(\omega)$ are the power spectra of $x(t)$ and $y(t)$, respectively. $S_{x y}(\omega)$ is the Fourier transform of the cross-correlation function [58],

$$
S_{x y}(\omega)=\frac{1}{2 \pi} \int_{-\infty}^{+\infty} C_{x y}(t) e^{-i \omega t} d t
$$

It is seen straightforwardly that $\operatorname{Coh}(\omega)$ satisfy

$$
0 \leqq \operatorname{Coh}(\omega) \leqq 1
$$

The coherence of fully correlated signals will be unity while that of uncorrelated random signals will vanish.

The phase spectrum is defined as the phase angle of $S_{x y}(\omega)$ on the complex plane by

$$
\Theta(\omega)=\tan ^{-1}\left(\frac{\operatorname{Im} S_{x y}(\omega)}{\operatorname{Re} S_{x y}(\omega)}\right),
$$

where Re and Im denote the real and imaginary parts. This is equal to the difference of the phase angles of the Fourier transforms of $x(t)$ and $y(t)$.

For each curve in Figure 6, $x(t)$ and $y(t)$ correspond to the energy gap coordinate $\Delta V_{\mathrm{LMCT}}$-ground and one of the structural parameters.
[1] Gray, H. B.; Solomon, E. I. In Copper Proteins; Spiro, T. G., Ed.; Wiley: New York, 1981.

[2] Sykes, A. G. Adv. Inorg. Chem. 1990, 36, 377

[3] Solomon, E. I.; Baldwin, M. J.; Lowery, M. D. Chem. Rev. 1992, 92, 521.

[4] Chowdhury, A.; Peteanu, L. A.; Holland, P. L.; Tolman, W. B. J. Phys. Chem. B 2002, 106, 3007.

[5] Fraga, E.; Webb, M. A.; Loppnow, G. R. J. Phys. Chem. 1996, $1000,3278$.

[6] Webb, M. A.; Loppnow, G. R. J. Phys. Chem. B 2002, 106, 2102.

[7] Edington, M. D.; Diffey, W. M.; Doria, W. J.; Riter, R. E.; Beck, W. F. Chem. Phys. Lett. 1997, 275, 119.

[8] Book, L. D.; Arnett, D. C.; Hu, H.; Scherer, N. F. J. Phys. Chem. A 1998, 102, 4350.

[9] Nakashima, S.; Nagasawa, Y.; Seike, K.; Okada, T.; Sato, M.; Kohzuma, T. Chem. Phys. Lett. 2000, 331, 396.

[10] Larsson, S.; Broo, A.; Sjölin, L. J. Phys. Chem. 1995, 99, 4860.

[11] Ryde, U.; Olsson, M. H.; Pierloot, K.; Roos, B. O. J. Mol. Biol. 1996, 261, 586.

[12] Ungar, L. W.; Scherer, N. F.; Voth, G. A. Biophys. J. 1997, 72, 5.

[13] Pierloot, K.; Kerpel, J. O. A. D.; Ryde, U.; Olsson, M. H. M.; Roos, B. O. J. Am. Chem. Soc. 1998, 120, 13156.

[14] Bizzarri, A. R.; Cannistraro, S. Physica A 1999, 267, 257.

[15] Lockwood, D. M.; Cheng, Y. K.; Rossky, P. J. Chem. Phys. Lett. 2001, 345, 159.

[16] Kawatsu, T.; Kakitani, T.; Yamato, T. J. Phys. Chem. B 2002, 106, 11356.

[17] Kobayashi, C.; Baldridge, K.; Onuchic, J. N. J. Chem. Phys. 2003, 119, 3550 .

[18] Olsson, M. H. M.; Hong, G.; Warshel, A. J. Am. Chem. Soc. 2003, 125, 5025 .

[19] Skourtis, S. S.; Balabin, I. A.; Kawatsu, T.; Beratan, D. N. Proc. Nat. Acad. Sci. USA 2005, 102, 3552.

[20] Sugiyama, A.; Takamatsu, Y.; Nishikawa, K.; Nagao, H.; Nishikawa, K. Int. J. Quant. Chem. 2006, 106, 3071.

[21] Ando, K. J. Phys. Chem. B 2004, 108, 3940.

[22] We are thus inclined to call it a 'ligand-bond to metal charge- transfer' (LBMCT), but in this paper we follow the convention to call it a LMCT.

[23] Santer, M.; Manthe, U.; Stock, G. J. Chem. Phys. 2001, 114, 2001.

[24] Ando, K.; Santer, M. J. Chem. Phys. 2003, 118, 10399.

[25] Cornell, W. D.; Cieplak, P.; Bayly, C. I.; Gould, I. R.; Jr, K. M. M.; Ferguson, D. M.; Spellmeyer, D. C.; Fox, T.; Caldwell, J. W.; Kollman, P. A. J. Am. Chem. Soc. 1995, 117, 5179.

[26] Dang, L. X.; Pettitt, B. M. J. Phys. Chem. 1987, 91, 3349.

[27] Inoue, T.; Sugawara, H.; Hamanaka, S.; Tsukui, H.; Suzuki, E.; Kohzuma, T.; Kai, Y. Biochem. 1999, 38, 6063.

[28] Tuckerman, M.; Berne, B. J.; Martyna, G. J. J. Chem. Phys. 1992, 97, 1990.

[29] Nosé, S. Prog. Theor. Phys. Suppl. 1991, 103, 1.

[30] Komeiji, Y.; Uebayasi, M.; Takata, R.; Shimizu, A.; Itsukashi, K.; Taiji, M. J. Comp. Chem. 1997, 18, 1546.

[31] The spectra were calculated by the fast Fourier transformation, and were averaged over 194 overlapping segments of the 800 ps trajectory. Then, a triangular filter function, which averages five points around a central point with a triangular weight, was applied to smooth the spectra. The Welch window was applied prior to the fast Fourier transformation. For the coherence spectra in Figure 6, the Parzen window and a nine points filter were used instead.

[32] Allen, M. P.; Tidesley, D. J Computer Simulation of Liquids: Clarendon: Oxford, 1987.

[33] The 800 ps trajectory was divided into 800 segments of 1 ps length, from which configurations were randomly sampled in every two segments.

[34] Kubo, R.; Toyozawa, Y. Prog. Theor. Phys. 1955, 13, 160.

[35] Hwang, J. K.; Warshel, A. Chem. Phys. Lett. 1997, 271, 223225.

[36] Borgis, D.; Lee, S.; Hynes, J. T. Chem. Phys. Lett. 1989, 162, 19.

[37] Ando, K. J. Chem. Phys. 1997, 107, 4585.

[38] The Fourier transforms of the TCFs are computed in practice from the power spectra of trajectories via the Wiener-Khinchin theorem.

[39] Normalized TCF for a parameter $A(t)$ is defined by $C(t)=$ 
$\langle\delta A(0) \delta A(t)\rangle_{0} /\left\langle\delta A(0)^{2}\right\rangle_{0}$, where $\delta A(t) \equiv A(t)-\langle A\rangle_{0}$.

[40] Hino, M. Spectral Analysis (in Japanese); Asakura: Tokyo, 1977.

[41] von Storch, H.; Zwiers, F. W. Statistical Analysis in Climate Research; Cambridge: Cambridge, 1999.

[42] It was found that $\left|\operatorname{Im} S_{x y}(\omega)\right|$ were so small that the deviations of $|\Theta(\omega)|$ from 0 or $\pi$ were smaller than $1 \times 10^{-5} \mathrm{rad}$. for all the data.

[43] In practical calculations, $\langle A(\infty)\rangle_{\text {neq }}$ were evaluated from averages in 3.6-4.0 ps region where the relaxations were observed to have converged.

[44] Burghardt, I.; Hynes, J. T. J. Phys. Chem. A 2006, 110, 11411.

[45] Riga, J. M.; Martens, C. C. J. Chem. Phys. 2004, 120, 6863.

[46] Roman, E.; Martens, C. C. J. Chem. Phys. 2004, 121, 11572.

[47] Jasper, A. W.; Truhlar, D. G. J. Chem. Phys. 2005, 122, 044101.

[48] Granucci, G.; Persico, M. J. Chem. Phys. 2007, 126, 134114.

[49] Tully, J. C. J. Chem. Phys. 1990, 93, 1061.

[50] Classical and Quantum Dynamics in Condensed Phase Simulations; Berne, B. J.; Ciccotti, G.; Coker, D., Eds.; World
Scientific: Singapore, 1998.

[51] Ando, K.; Sumi, H. J. Phys. Chem. B 1998, 102, 10991.

[52] Hayashi, S.; Tajkhorshid, E.; Schulten, K. Biophys. J. 2003, 85, 1440.

[53] Olsen, S.; Toniolo, A.; Ko, C.; Manohar, L.; Lamothe, K.; Martinez, T. J. In Computational Photochemistry; Olivucci, M., Ed.; Elsevier: Amsterdam, 2005.

[54] Smith, P. E.; Pettitt, B. M. J. Chem. Phys. 1991, 95, 8430.

[55] Dang, L. X. J. Chem. Phys. 1992, 97, 1919.

[56] Rozanska, X.; Chipot, C. J. Chem. Phys. 2000, 112, 9691.

[57] Fennell, C. J.; Gezelter, J. D. J. Chem. Phys. 2006, 124, 234104.

[58] In practice we calculated $S_{x y}(\omega)$ from the Fourier transforms of $x(t)$ and $y(t)$ via

$$
S_{x y}(\omega)=\frac{2 \pi}{T} X(\omega)^{*} Y(\omega)
$$

in which $T$ is the total length of the time record. 


\title{
Supporting Information for
}

\section{Ligand-to-metal charge-transfer dynamics in a blue copper protein plastocyanin: A molecular dynamics study}

\author{
Koji Ando
}

Department of Chemistry, Graduate School of Science, Kyoto University,

Sakyo-ku, Kyoto 606-8502, Japan

Table S1. Atomic charge parameters for the Cu-cysteine moiety.

\begin{tabular}{lrrrrrrr}
\hline & \multicolumn{7}{c}{ state } \\
\cline { 2 - 8 } atom & \multicolumn{1}{c}{0} & \multicolumn{1}{c}{1} & \multicolumn{1}{c}{2} & \multicolumn{1}{c}{3} & \multicolumn{1}{c}{4} & \multicolumn{1}{c}{5} & \multicolumn{1}{c}{6} \\
\hline $\mathrm{N}$ & -0.4157 & -0.4157 & -0.4157 & -0.4157 & -0.4157 & -0.4157 & -0.4157 \\
$\mathrm{H}_{\mathrm{N}}$ & 0.2719 & 0.2719 & 0.2719 & 0.2719 & 0.2719 & 0.2719 & 0.2719 \\
$\mathrm{C}_{\alpha}$ & 0.3023 & 0.3017 & 0.3008 & 0.3008 & 0.3012 & 0.3025 & 0.3033 \\
$\mathrm{H}_{\alpha}$ & 0.0766 & 0.0766 & 0.0766 & 0.0766 & 0.0766 & 0.0766 & 0.0766 \\
$\mathrm{C}_{\beta}$ & -0.7862 & -0.7785 & -0.7772 & -0.7775 & -0.7744 & -0.8223 & -0.8439 \\
$\mathrm{H}_{\beta}$ & 0.2645 & 0.2638 & 0.2631 & 0.2629 & 0.2634 & 0.2647 & 0.2654 \\
$\mathrm{~S}_{\gamma}$ & -0.4984 & -0.5614 & -0.5733 & -0.5713 & -0.5901 & -0.0294 & 0.1567 \\
$\mathrm{C}$ & 0.5973 & 0.5973 & 0.5973 & 0.5973 & 0.5973 & 0.5973 & 0.5973 \\
$\mathrm{O}$ & -0.5679 & -0.5679 & -0.5679 & -0.5679 & -0.5679 & -0.5679 & -0.5679 \\
$\mathrm{Cu}$ & 1.4911 & 1.5484 & 1.5613 & 1.5600 & 1.5743 & 1.0576 & 0.8909 \\
\hline
\end{tabular}

Table S2. Atomic charge parameters for the histidine and methionine ligands.

\begin{tabular}{lrlr}
\hline \multicolumn{2}{c}{ histidine } & \multicolumn{2}{c}{ methionine } \\
\hline $\mathrm{N}$ & -0.4157 & $\mathrm{~N}$ & -0.4157 \\
$\mathrm{H}_{\mathrm{N}}$ & 0.2719 & $\mathrm{H}_{\mathrm{N}}$ & 0.2719 \\
$\mathrm{C}_{\alpha}$ & -0.0581 & $\mathrm{C}_{\alpha}$ & -0.0237 \\
$\mathrm{H}_{\alpha}$ & 0.1360 & $\mathrm{H}_{\alpha}$ & 0.0880 \\
$\mathrm{C}_{\beta}$ & -0.0294 & $\mathrm{C}_{\beta}$ & 0.2455 \\
$\mathrm{H}_{\beta}$ & 0.1457 & $\mathrm{H}_{\beta}$ & 0.0241 \\
$\mathrm{C}_{\gamma}$ & -0.0908 & $\mathrm{C}_{\gamma}$ & -0.8179 \\
$\mathrm{~N}_{\delta}$ & -0.6781 & $\mathrm{H}_{\gamma}$ & 0.2436 \\
$\mathrm{C}_{\epsilon}$ & 0.1964 & $\mathrm{~S}_{\delta}$ & 0.1615 \\
$\mathrm{H}_{\epsilon}$ & 0.2388 & $\mathrm{C}_{\epsilon}$ & -0.8331 \\
$\mathrm{~N}_{\epsilon}$ & -0.5225 & $\mathrm{H}_{\epsilon}$ & 0.2529 \\
$\mathrm{H}_{\epsilon}$ & 0.4546 & $\mathrm{C}$ & 0.5973 \\
$\mathrm{C}_{\delta}$ & -0.0713 & $\mathrm{O}$ & -0.5679 \\
$\mathrm{H}_{\delta}$ & 0.2474 & & \\
$\mathrm{C}$ & 0.5973 & & \\
$\mathrm{O}$ & -0.5679 & & \\
\hline & & &
\end{tabular}




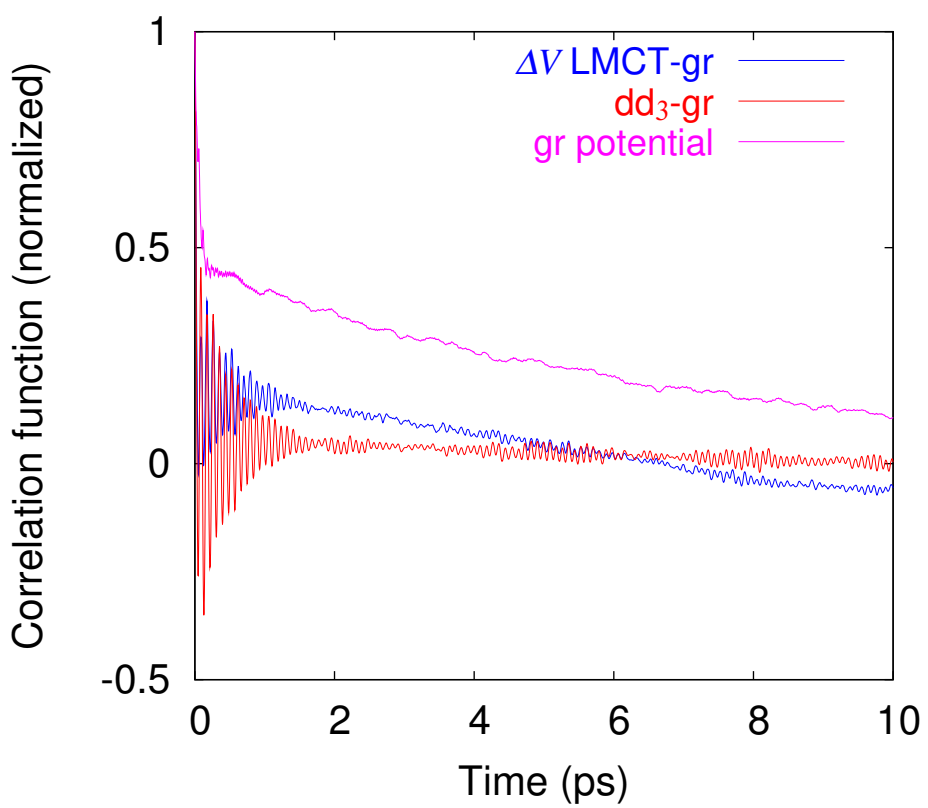

Figure S1. Time correlation functions of the energy gap coordinates corresponding to Figure $3 \mathrm{a}$ but in the longer time range. The function for the ground state potential energy is included for comparison. 

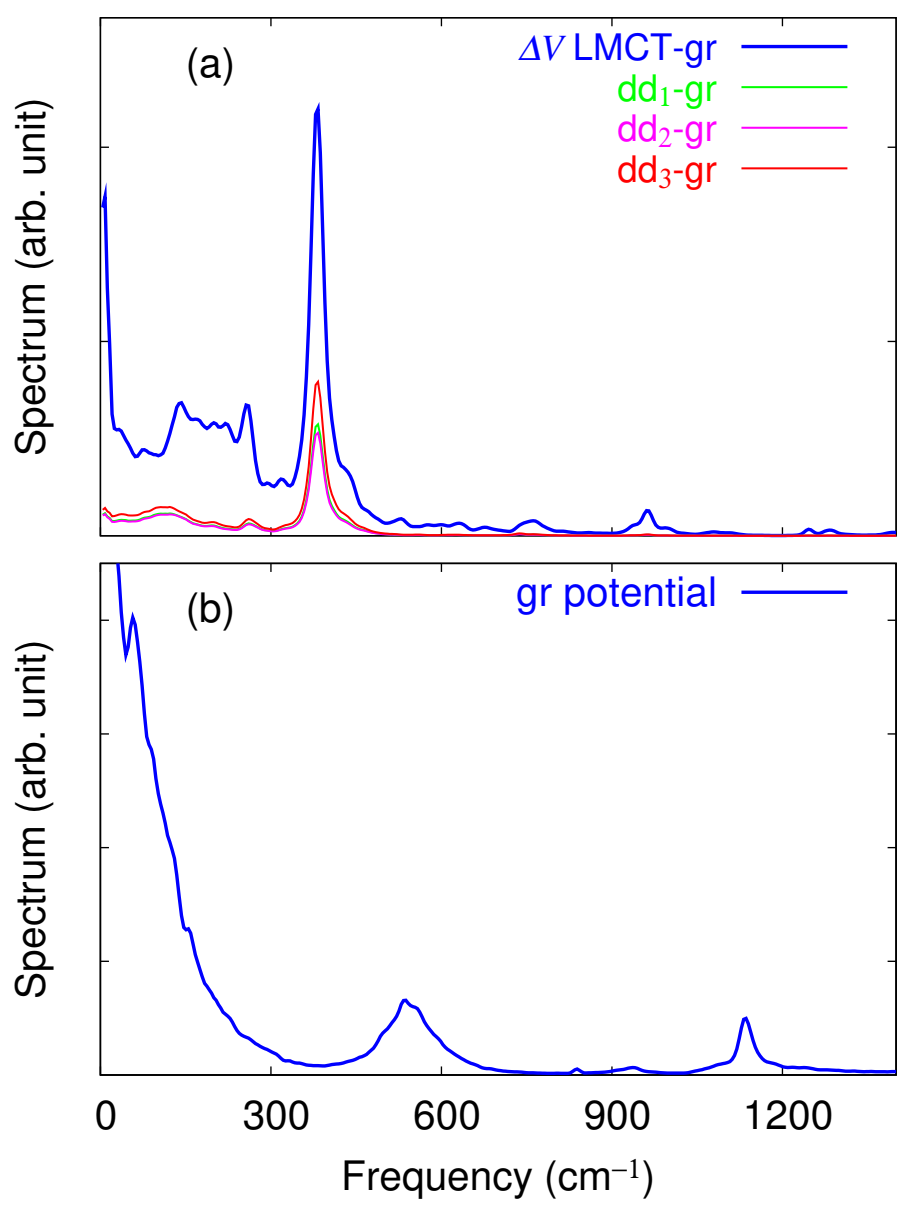

Figure S2. Power spectra of the energy gap coordinates corresponding to Figure $3 b$ but to the higher frequency range (a). The spectrum for the ground state potential energy (b). 

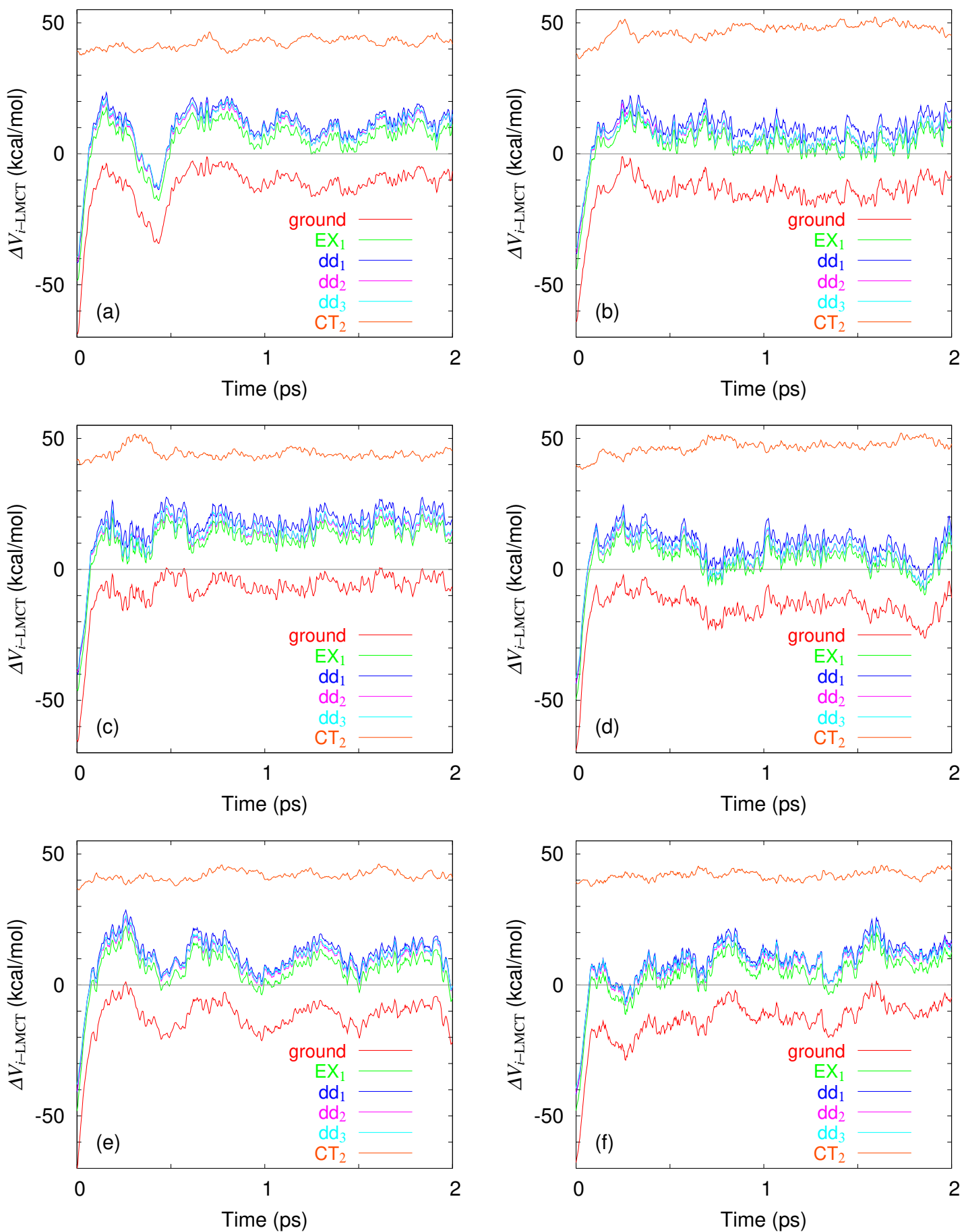

Figure S3. Randomly selected non-equilibrium trajectories corresponding to Figure 7b. 

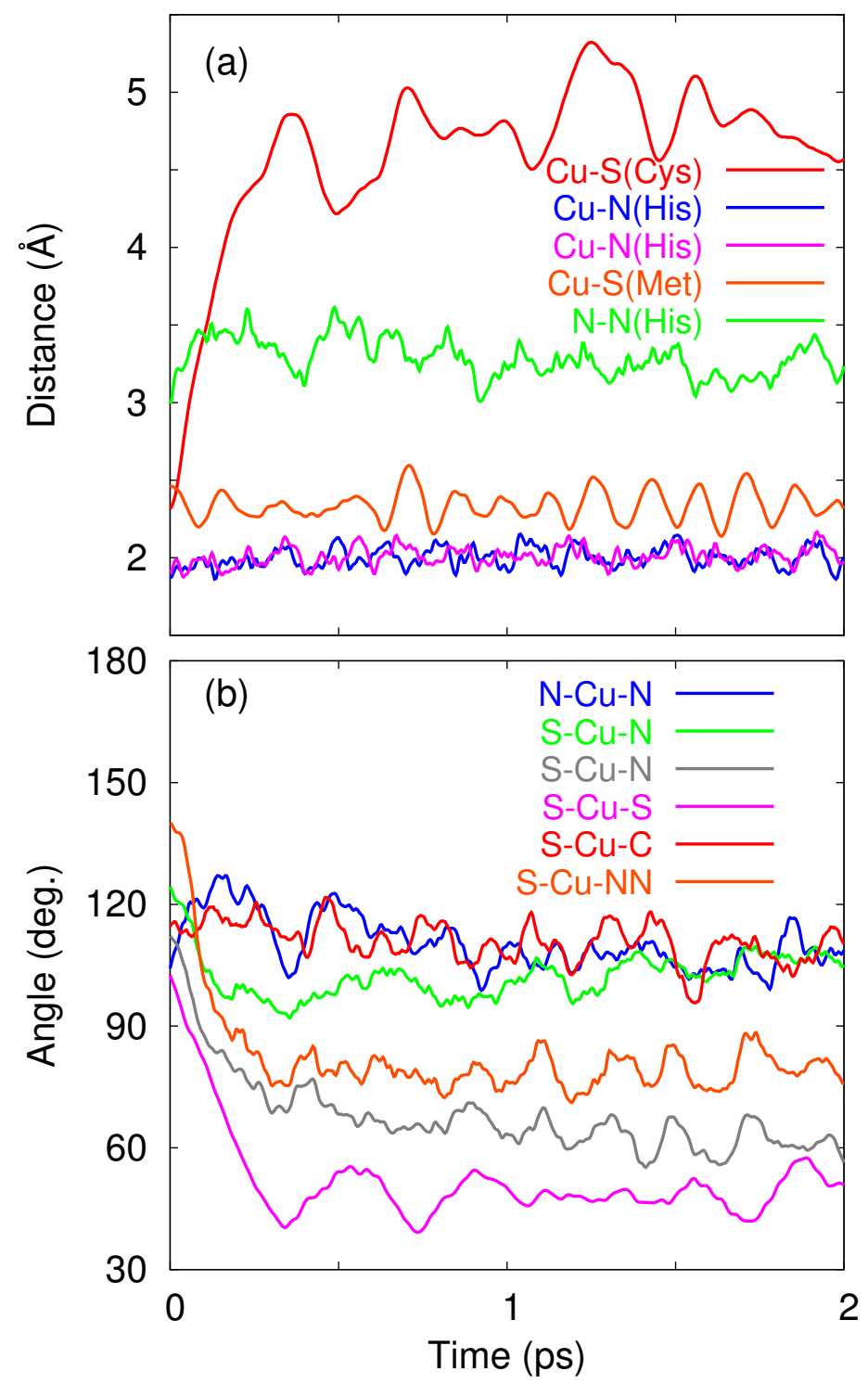

Figure S4. Non-equilibrium dynamics of the structural parameters, corresponding to Figure 8 but from a representative single trajectory. 